Bangladesh J. Plant Taxon. 17(1): 33-53, 2010 (June)

(C) 2010 Bangladesh Association of Plant Taxonomists

\title{
ANGIOSPERMIC FLORA OF RUNCTIA SAL FOREST, BANGLADESH. II. MAGNOLIOPSIDA (DICOTS)
}

\author{
Ershad Tutul*, Md. Zashim Uddin, Md. Oliur Rahman and Md. Abul Hassan \\ Department of Botany, University of Dhaka, Dhaka 1000, Bangladesh
}

Keywords: Angiosperms; Magnoliopsida; Runctia sal forest.

\begin{abstract}
This paper deals with a total of 153 plant species under 120 genera and 52 families of the division Magnoliopsida (Dicots) of the Runctia Sal forest in Sherpur district. Habit analysis shows that herbs are represented by 34 , shrubs 36 , trees 65 , climbers 17 and epiphyte by a single species. Updated nomenclature, habit, habitat and representative specimen have been furnished under each taxon.
\end{abstract}

\section{Introduction}

The Runctia sal forest is a deciduous type of forest with an area of 3363.93 ha and consists of many hills and hillocks. The forest is interspersed by hills, valleys, streams and cultivated rice fields. The forest consists of three beats, namely, Runctia, Gazni and Tawakocha. Despite Runctia sal forest plays a significant role in the local economy, environment and traditional health care system, very little is known about the flora of the forest. Very recently Tutul et al. (2009) have described 49 species under Liliopsida (Monocots) from the same forest for the first time. The present paper is the continuation of the previous one. The present study has been undertaken in order to identify the species under Magnoliopsida (Dicots). The detailed information about the forest has been provided in Tutul et al. (2009).

\section{Materials and Methods}

Materials and methods of the work carried out were also provided in the previous paper (Tutul et al. 2009). The specimens were collected from hill tops, hill slopes, forest floors, forest margins, streams, swamps and plain lands of the forest area. They were identified and the identification were confirmed with the help of Hooker (1872-1888), Prain (1903), Kanjilal et al. (1934, 1938, 1939, 1940), Dassanayake and Fosberg (19801985), Khan (1972-1987), Khan and Rahman (1989-2002), Hassan (1996), Hajra et al. (1997) and Singh et al. (2000). The families were arranged according to the Cronquist (1981) and the genera and species under each family were placed alphabetically. Only one representative specimen was cited under each species because of page limitation. The specimens were deposited in the Dhaka University Herbarium (DUH), presently known as Salar Khan Herbrium.

*Corresponding author. E-mail: tutul_ershad@yahoo.com 


\section{Systematic enumeration}

A total of 153 dicot species were recorded from the Runctia Sal forest. The identified species were assigned to 120 genera and 52 families. Habit analysis showed that herbs were represented by 34, shrubs 36, trees 65, climbers 17 and epiphyte by a single species. Species representation in the families varied from 1 to 15 . The family Rubiaceae appears to be the largest represented by 15 species followed by Euphorbiaceae (12), Fabaceae (10), Moraceae (7), Caesalpiniacae (7), Asteraceae (7) and Verbenaceae (6). Shorea robusta Roxb. ex Gaertn. f. is the most dominant species in the forest. Some of the other dominant species of the forest include: Lagerstroemia indica L., Mussaenda frondosa L., Grewia nervosa (Lour.) Panigr., Melastoma melabathricum L., Clerodendrum viscosum Vent., Morinda angustifolia Roxb., Mikania cordata (Burm. f.) Robinson etc.

\section{Magnoliaceae}

Michelia champaca L., Sp. Pl.: 536 (1753). Michelia rheedii Wight (1840). Local name: Champa.

A semi-evergreen to evergreen tree. On the hill slope. Representative specimen: Gazni, 18.05.2007, Ershad Tutul 181 (DUH).

\section{Annonaceae}

Miliusa velutina (Dunal) Hook. f. \& Thom., Fl. Ind.: 139 (1855). Uvaria villosa Roxb. (1832). Local name: Gandhi gajari.

A deciduous tree. Top of the hill. Representative specimen: Gazni, 17.05.2008, Ershad Tutul 392 (DUH).

\section{Lauraceae}

Litsea angustifolia Wall. ex Hook. f., Fl. Brit. Ind. 5: 169 (1886). Tetranthera saligna Nees (1831). Local name: Haria.

A bushy evergreen shrub. On the hill slope. Representative specimen: Gazni, 18.05.2007, Ershad Tutul 238 (DUH).

Litsea glutinosa (Lour.) Robinson, Philip. J. Sci. Bot. 6: 321 (1911). Litsea chinensis Lamk. (1792). Local name: Kukurchita.

A small to medium-sized evergreen tree. On the edges of forest. Representative specimen: Gazni, 18.05.2007, Ershad Tutul 182 (DUH).

Litsea monopetala (Roxb.) Pers., Syn. Pl. 2: 4 (1807). Tetranthera monopetala Roxb. (1798). Local name: Akorma.

A medium-sized tree. On the hill slope. Representative specimen: Gazni, 16.05.2007, Ershad Tutul 101 (DUH). 


\section{Menispermaceae}

Cyclea barbata Miers, Contrib. Bot. 3: 237 (1871). Cyclea wallichii Diels (1910).

A long slender climber. On the hill slope. Representative specimen: Gazni, 18.05.2007, Ershad Tutul 185 (DUH).

\section{Ulmaceae}

Trema orientalis (L.) Blume, Ann. Mus. Bot. Lugd.-Bat. 2: 62 (1856). Sponia orientalis (L.) Decne. (1834). Local name: Jibon.

A small to medium-sized tree. Near the swamps of the forest. Representative specimen: Tawakocha, 28.10.2007, Ershad Tutul 273 (DUH).

\section{Moraceae}

Artocarpus chama Buch.-Ham. ex Wall., Cat. no. 4657 (1814). Artocarpus chaplasha Roxb. (1832). Local name: Chamkathal.

A large deciduous tree. On the hill top. Representative specimen: Tawakocha, 28.10.2007, Ershad Tutul 275 (DUH).

Artocarpus heterophyllus Lamk., Encycl. Meth. 3: 210 (1789). Artocarpus philippensis Lamk. (1789). Local name: Kanthal.

An evergreen tree. On hill top and slope. Representative specimen: Gazni, 18.05.2007, Ershad Tutul 237 (DUH).

Artocarpus lacucha Hook. f., Fl. Brit. Ind. 3: 524 (1832). Local name: Dewa.

A deciduous tree. On the hill top and slope. Representative specimen: Gazni, 16.05.2007, Ershad Tutul 67 (DUH).

Ficus benghalensis L., Sp. Pl.: 1059 (1753). Ficus indica L. (1753). Local name: Bot.

A large, evergreen tree. On the hill slope and edges of forest. Representative specimen: Gazni, 18.05.2007, Ershad Tutul 239 (DUH).

Ficus virens Aiton, Hort. Kew. 3: 451 (1789). Ficus infectoria Roxb. (1814). Local name: Pakur.

A deciduous or semi-deciduous tree. On the top hill. Representative specimen: Runctia, 17.05.2007, Ershad Tutul 114 (DUH).

Ficus racemosa L., Sp. Pl.: 1060 (1753). Ficus goolerea Roxb. (1832). Local name: Dumur.

A medium-sized evergreen tree. On the edges of forest. Representative specimen: Gazni, 16.05.2007, Ershad Tutul 97 (DUH).

Streblus asper Lour., Fl. Cochinch. 2: 615 (1790). Streblus lactescens Blume (1918). Local name: Sheora. 
A bushy evergreen tree. On the forest edges. Representative specimen: Runctia, 17.05.2007, Ershad Tutul 123 (DUH).

\section{Amaranthaceae}

Amaranthus spinosus L., Sp. Pl. 1: 991 (1753). Local name: Kanta-note.

An annual, erect herb. On waste lands, roadsides, fields and gardens. Representative specimen: Runctia, 17.05.2007, Ershad Tutul 127 (DUH).

Cyathula prostrata (L.) Blume, Bijdr.: 549 (1825). Achyranthes prostrata L. (1762).

A slender, annual herb. On the edges of forest and shady areas. Representative specimen: Tawakocha, 28.10.2007, Ershad Tutul 244 (DUH).

\section{Polygonaceae}

Persicaria stagnina (Hamilt. ex Meissn.) Hassan, Bangladesh J. Plant. Taxon. 3(1): 81 (1996). Polygonum stagninum Hamilton ex Meissn. (1832). Local name: Bara bishkathali.

A perennial herb. On the forest edges. Representative specimen: Gazni, 15.05.2007, Ershad Tutul 24 (DUH).

\section{Dilleniaceae}

Dillenia indica L., Sp. Pl. 1: 535 (1753). Dillenia speciosa Thunb. (1791). Local name: Chalta.

A medium-sized to large evergreen tree. On the hill slope of the forest. Representative specimen: Runctia, 16.05.2007, Ershad Tutul 73 (DUH).

Dillenia pentagyna Roxb., Pl. Corom. 1: 21, t. 20 (1795). Dillenia baillonii Pierre ex Lanessan (1886). Local name: Bon chalta.

A large, deciduous tree. On the top hill. Representative specimen: Gazni, 16.05.2007, Ershad Tutul 103 (DUH).

\section{Dipterocarpaceae}

Shorea robusta Roxb. ex Gaertn. f., De Fruct. 3: 48 (1805). Local name: Gozari.

A large, semi-deciduous tree. On the hill top. Representative specimen: Gazni, 15.05.2007, Ershad Tutul 26 (DUH).

\section{Theaceae}

Schima wallichii (DC.) Korth. in Temminck, Verh. Nat. Gesch. Bot. 3: 143 (1840). Schima brevipes Craib (1915).

A large evergreen tree. Common on the hill top. Representative specimen: Gazni, 16.05.2007, Ershad Tutul 72 (DUH). 


\section{Clusiaceae}

Garcinia cowa Roxb. ex DC., Prodr. 1: 561 (1824). Garcinia roxburghii Wight (1840). Local name: Kao.

A dioecious, tall tree. On the hill slope. Representative specimen: Gazni, 17.05.2008, Ershad Tutul 339 (DUH).

\section{Tiliaceae}

Grewia nervosa (Lour.) Panigr., Taxon 34: 702 (1985). Grewia microcos L. (1767). Local name: Asar.

A semi-deciduous tree. Very common on the hill slope. Representative specimen: Gazni, 15.05.2007, Ershad Tutul 12 (DUH).

Triumfetta pentandra A. Rich. in Guill. \& Perr., Fl. Senegamb. Tent. 1: 93, t. 19 (1831). Triumfetta rhomboidea Jacq. var. pentandra (A. Rich.) J. L. Ellis (1983).

An annual herb. On the forest edges. Representative specimen: Tawakocha, 28.10.2007, Ershad Tutul 246 (DUH).

\section{Sterculiaceae}

Abroma augusta (L.) L. f., Suppl.: 341 (1781). Abroma mollis DC. (1824). Local name: Ulatkambol.

A shrub or small tree. On the hill slope. Representative specimen: Gazni, 18.05.2007, Ershad Tutul 161 (DUH).

Sterculia villosa Roxb. ex Smith in Rees, Cycl. 34, no. 16 (1816). Sterculia armata Mast. in Hook. f. (1874). Local name: Udal.

A medium-sized tree. On hill slope. Representative specimen: Runctia, 17.05.2007, Ershad Tutul 147 (DUH).

\section{Malvaceae}

Sida cordata (Burm. f.) Borss., Blumea 14: 182 (1966). Melochia cordata Burm. f. (1768). Local name: Junka.

An annual herb. On the forest edges. Representative specimen: Gazni, 15.05.2007, Ershad Tutul 35 (DUH).

Sida rhombifolia L., Sp. Pl.: 684 (1753). Sida retusa L. (1763). Local name: Lal berela. An undershrub. On the forest edges. Representative specimen: Tawakocha, 28.10.2007, Ershad Tutul 249 (DUH).

Thespesia lampas (Cav.) Dalz. \& Gibs., Bombay Fl.: 19 (1861). Hibiscus lampas Cav. (1787). Local name: Bonkarpas.

An erect, slightly branched shrub. On the hill slope. Representative specimen: Tawakocha, 28.10.2007, Ershad Tutul 263 (DUH). 
Urena lobata L., Sp. Pl.: 692 (1753). Urena palmata Roxb. (1832). Local name: Belaz. An undershrub. On the forest edges. Representative specimen: Gazni, 15.05.2007, Ershad Tutul 21 (DUH).

\section{Lecythidaceae}

Careya arborea Roxb., Pl. Corom. 3: 14 (1811). Local name: Kumvi.

A fire resistant, medium-sized tree. On the hill top. Representative specimen: Gazni, 18.05.2007, Ershad Tutul 184 (DUH).

\section{Flacourtiaceae}

Flacourtia jangomas (Lour.) Raeusch., Nom. Bot. ed. 3: 290 (1797). Flacourtia cataphracta Roxb. (1806). Local name: Paniala.

A small evergreen shrub. On the hill slope and forest edges. Representative specimen: Gazni, 17.05.2008, Ershad Tutul 371 (DUH).

\section{Cucurbitaceae}

Solena amplexicaulis (Lamk.) Gandhi in Saldanha \& Nicolson, Fl. Hassan Distr.: 179 (1976). Melothria heterophylla (Lour.) Cogn. (1881). Local name: Rakhal gota.

A perennial, climbing herb. On the hill slope. Representative specimen: Runctia, 16.05.2007, Ershad Tutul 63 (DUH).

Trichosanthes tricuspidata Lour., Fl. Cochinch.: 589 (1790). Trichosanthes bracteata (Lamk.) Voigt (1848). Local name: Makal phal.

A climber. On the forest edges. Representative specimen: Tawakocha, 28.10.2007, Ershad Tutul 274 (DUH).

\section{Capparaceae}

Crateva magna (Lour.) DC., Prodr. 1: 243 (1824). Crateva nurvala Buch.-Ham. (1827). Local name: Barun.

A small to medium-sized deciduous tree. Near the swamps and on edges of forest. Representative specimen: Gazni, 16.05.2007, Ershad Tutul 88 (DUH).

\section{Myrsinaceae}

Maesa indica (Roxb.) A. DC., Trans. Linn. Soc. 17: 134 (1834). Baeobotrys indica Roxb. (1814). A shrub or small tree. On the hill slope. Representative specimen: Runctia, 16.05.2007, Ershad Tutul 55 (DUH).

\section{Mimosaceae}

Acacia concinna (Willd.) DC., Prod. 2: 464 (825). Mimosa concinna Willd. (1806). Local name: Bonrita. 
A scandent and spiny climber. On the edges of forest. Representative specimen: Gazni, 17.05.2008, Ershad Tutul 387 (DUH).

Acacia mangium Willd., Sp. Pl. ed. 4, 4: 1053 (1806). Racosperma mangium (Willd.) Pedley (1987). Local name: Mangium.

A fast growing, exotic tree. Planted on the forest bed. Representative specimen: Tawakocha, 28.10.2007, Ershad Tutul 269 (DUH).

Acacia auriculiformis A. Cunn. ex Benth. \& Hook., Lond. J. Bot. 1: 377 (1842). Acacia moniliformis Griseb.(1874). Local name: Akashmoni.

An exotic tree. Planted on the forest bed. Representative specimen: Gazni, 16.05.2007, Ershad Tutul 70 (DUH).

Mimosa pudica L., Sp. Pl. 1: 518 (1753). Mimosa asperata Blanco (1837). Local name: Lajjabati.

An annual or perennial herb. On the edges of forest. Representative specimen: Gazni, 15.05.2007, Ershad Tutul 16 (DUH).

Samanea saman (Jacq.) Merr., J. Wash. Acad. Sci. 6: 47 (1916). Enterolobium saman Jacq. (1897). Local name: Rendi.

A very large tree. On hill top and along the roadsides. Representative specimen: Tawakocha, 28.10.2007, Ershad Tutul 277 (DUH).

\section{Caesalpiniaceae}

Bauhinia purpurea L., Sp. Pl. 1: 375 (1753). Phanera parpurea (L.) Benth. (1852). Local name: Sada kanchon.

A small deciduous tree. On hill slope and forest edges. Representative specimen: Runctia, 16.05.2007, Ershad Tutul 74 (DUH).

Bauhinia scandens L., Sp. Pl. 1: 344 (1753). Lasiobema horsfieldii Miq. (1855). Local name: Kanchon.

A woody climber. On the forest edges. Representative specimen: Gazni, 16.05.2007, Ershad Tutul 108 (DUH).

Caesalpinia crista L., Sp. Pl. 1: 380 (1753). Caesalpinia chinensis Roxb. (1832). Local name: Letkanta.

A prickly shrub or small tree. On the edges and near the swamps of forest. Representative specimen: Gazni, 28.10.2007, Ershad Tutul 287 (DUH).

Caesalpinia cucullata Roxb., Fl. Ind. ed. 2, 2: 358 (1832). Mezoneuron cucullatum (Roxb.) Wight \& Arn. (1834).

A large scandent shrub. Near the swamps of forest. Representative specimen: Gazni, 18.05.2007, Ershad Tutul 155 (DUH). 
Caesalpinia digyna Rottler, Ges. Naturf. Freunde. Berlin. Schrift. 4: 200, t. 3 (1803). Caesalpinia oleosperma Roxb. (1832).

A large shrub. On the hill slope and edges of forest. Representative specimen: Runctia, 16.05.2007, Ershad Tutul 56 (DUH).

Cassia fistula L., Sp. Pl. 1: 377 (1753). Cassia rhombifolia Roxb. (1832). Local name: Bandorlathi.

A deciduous, medium-sized tree. On hill slope of forest. Representative specimen: Gazni, 17.05.2008, Ershad Tutul 368 (DUH).

Senna tora (L.) Roxb., Fl. Ind. 2: 340 (1832). Cassia tora L. (1753). Local name: Kalkesunda.

A perennial herb. On the forest edges. Representative specimen: Gazni, 16.05.2007, Ershad Tutul 98 (DUH).

\section{Fabaceae}

Abrus precatorius L., Syst. Nat. ed. 12: 472 (1767). Glycine abrus L. (1753). Local name: Rati.

A perennial climber. On the forest edges. Representative specimen: Gazni, 16.05.2007, Ershad Tutul 40 (DUH).

Crotalaria pallida Ait., Hort. Kew. 3: 20 (1789). Crotalaria saltiana auct. non Andr. (1811). Local name: Jhunjhuni.

An erect, annual herb. On the edges of forest. Representative specimen: Gazni, 15.05.2007, Ershad Tutul 22 (DUH).

Dalbergia stipulacea Roxb., Fl. Ind. 3: 233 (1814). Dalbergia ferruginea Roxb. (1832).

A woody climber. On the hill top. Representative specimen: Tawakocha, 28.10.2007, Ershad Tutul 287 (DUH).

Derris elegans Benth. var. vestita (Baker) Prain, J. Asiat. Soc. Beng. 66: 103 (1897). Derris vestita Baker (1878).

A woody climber. On the hill slope. Representative specimen: Gazni, 18.05.2007, Ershad Tutul 222 (DUH).

Erythrina fusca Lour., Fl. Cochinch.: 427 (1790). Erythrina ovalifolia Roxb. (1832). Local name: Mandar.

A small, soft wooded, deciduous tree. On the edges of forest. Representative specimen: Tawakocha, 28.10.2007, Ershad Tutul 270 (DUH).

Erythrina variegata L., Diss. Herb. Amb. Amoen. Acad. 4: 122 (1754). Erythrina indica Lamk. (1786). Local name: Mandar.

A small to medium-sized, deciduous tree. On the forest edges. Representative specimen: Gazni, 16.05.2007, Ershad Tutul 104 (DUH). 
Mucuna pruriens (L.) DC., Prodr. 2: 405 (1825). Mucuna prurita Hook. f. (1831). Local name: Alkushi.

An annual or perennial climbing herb. On the hill slope. Representative specimen: Gazni, 16.05.2008, Ershad Tutul 331 (DUH).

Tephrosia candida DC., Prodr. 2: 249 (1825). Robinia candida Roxb. (1832).

A small branched shrub. On the hill slope. Representative specimen: Gazni, 18.05.2007, Ershad Tutul 186 (DUH).

Uraria crinita (L.) Desv. ex DC., Prodr. 2: 324 (1825). Hedysarum crinitum L. (1876). A shrub. On hilly forest areas. Representative specimen: Gazni, 18.05.2007, Ershad Tutul 223 (DUH).

Vigna pilosa (Willd.) Baker in Hook. f., Fl. Brit. Ind. 2: 207 (1879). Dolichos pilosum Willd. (1777).

An annual herb. On hilly areas. Representative specimen: Gazni, 16.05.2007, Ershad Tutul 45 (DUH).

\section{Lythraceae}

Lagerstroemia parviflora Roxb. var. benghalensis C. B. Clarke in Hook. f., Fl. Brit. Ind. 2: 576 (1879). Local name: Sidha.

A tree with white flowers. Very common on the hill top. Representative specimen: Gazni, 16.05.2008, Ershad Tutul 301 (DUH).

Lagerstroemia indica L., Sp. Pl. ed. 2, 1: 734 (1762). Lagerstroemia chinensis Lamk. (1789). Local name: Choto jarul.

A small tree. On forest edges. Representative specimen: Gazni, 28.10.2007, Ershad Tutul 252 (DUH).

Lagerstroemia macrocarpa Wall., Cat. no. 2114 (1831). Local name: Ban jarul.

A medium-sized tree. Occurs in mixed and open forest. Representative specimen: Gazni, 17.05.2007, Ershad Tutul 112 (DUH).

Lagerstroemia speciosa (L.) Pers., Syn. 2: 72 (1807). Lagerstroemia regina Roxb. (1832). Local name: Jarul.

A large, much-branched, deciduous tree. On the forest edges. Representative specimen: Gazni, 17.05.2008, Ershad Tutul 356 (DUH).

\section{Myrtaceae}

Eucalyptus grandis Hill ex Maiden, J. R. Soc. N. S. W. 52: 50 (1980). Eucalyptus saligna var. pallidivalvis Baker \& Smith (1902). Local name: Eucalyptus.

A large tall tree. On the forest edges. Representative specimen: Gazni, 18.05.2007, Ershad Tutul 235 (DUH). 
Syzygium fruticosum DC., Prodr. 3: 260 (1828). Eugenia fruticosa Roxb. (1832). Local name: Bon jam.

A large shrub to small tree. On hilly slope of forest. Representative specimen: Gazni, 17.05.2008, Ershad Tutul 378 (DUH).

\section{Onagraceae}

Ludwigia adscendens (L.) Hara, J. Jap. Bot. 28: 291 (1953). Jussiaea adscendens L. (1767). Local name: Molchi.

A rarely creeping to floating herb. Near the forest swamps. Representative specimen: Gazni, 15.05.2007, Ershad Tutul 30 (DUH).

\section{Melastomataceae}

Melastoma malabathricum L., Sp. Pl. 1: 390 (1753). Melastoma royenii Blume (1831). Local name: Motmoti.

An undershrub to shrub. Very common in forest margin. Representative specimen: Gazni, 15.05.2007, Ershad Tutul 15 (DUH).

\section{Combretaceae}

Terminalia arjuna (Roxb. ex DC.) Wight \& Arn., Prodr.: 314 (1834). Pentaptera arjuna Roxb. ex DC. (1828). Local name: Arjun.

A medium to large tree. On hill slope and edges of forest. Representative specimen: Gazni, 28.10.2007, Ershad Tutul 278 (DUH).

Terminalia catappa L., Syst. Nat. ed. 12: 674 (1767). Terminalia procera Roxb. (1832). Local name: Katbadam.

A medium to large deciduous tree. On the forest edges. Representative specimen: Runctia, 17.05.2007, Ershad Tutul 118 (DUH).

\section{Alangiaceae}

Alangium chinense (Lour.) Harms in Ber. Deuts. B. Ges. 15: 24 (1897). Marlea begoniaefolia Roxb. (1814). Local name: Marleza gachh.

A small deciduous tree with grey bark. On hill slope. Representative specimen: Gazni, 16.05.2008, Ershad Tutul 321 (DUH).

\section{Loranthaceae}

Scurrula parasitica L., Sp. Pl.: 110 (1753). Loranthus scurrula L. (1762).

A stem parasite. On branches of woody angiosperms. Representative specimen: Tawakocha, 28.10.2007, Ershad Tutul 288 (DUH). 


\section{Euphorbiaceae}

Antidesma acuminatum Wall. in Wight, Icon. Pl. Ind. Or. 6: t. 199 (1853). Local name: Shial.

A small to large tree. On hill slope. Representative specimen: Gazni, 16.05.2007, Ershad Tutul 49 (DUH).

Bridelia stipularis (L.) Blume, Bijdr.: 597 (1825). Clutia stipularis L. (1753).

A large, more or less climbing shrub. On the forest edges. Representative specimen: Gazni, 28.10.2007, Ershad Tutul 258 (DUH).

Flueggea virosa (Roxb. ex Willd.) Baill., Etudes Gen. Euphorb.: 593 (1858). Phyllanthus virosus Roxb. ex Willd. (1805). Local name: Shikori.

A glabrous shrub or small tree. On the forest edges. Representative specimen: Gazni, 16.05.2008, Ershad Tutul 303 (DUH).

Jatropha gossypifolia L., Sp. Pl.: 1066 (1753). Local name: Lalvarenda.

A soft-wooded shrub. On open sunny places. Representative specimen: Gazni, 18.05.2007, Ershad Tutul 234 (DUH).

Macaranga denticulata (Blume) Muell.-Arg. in DC., Prodr. 15, 2: 1000 (1866). Mappa gummiflua Miq. (1858). Local name: Bura.

A medium-sized, evergreen tree. Hill slope of the forest. Representative specimen: Gazni, 16.05.2007, Ershad Tutul 96 (DUH).

Macaranga peltata (Roxb.) Muell.-Arg. in DC., Prodr. 15, 2: 1010 (1866). Osyris peltata Roxb. (1832).

A small evergreen tree. On the hill slope. Representative specimen: Gazni, 18.05.2007, Ershad Tutul 175 (DUH).

Mallotus tetracoccus (Roxb.) Kurz, For. Fl. Brit. Burma 2: 382 (1877). Mallotus albus (Roxb.) Muell.-Arg. (1865). Local name: Muralia.

A small tree. On the hill slope. Representative specimen: Gazni, 18.05.2007, Ershad Tutul 220 (DUH).

Manihot esculenta Crantz, Inst. 1: 167 (1766). Manihot edule A. Rich. (1853). Local name: Shimul alu.

A glabrous shrub with tuberous root. On the forest edge. Representative specimen: Gazni, 16.05.2007, Ershad Tutul 59 (DUH).

Phyllanthus emblica L., Sp. Pl.: 982 (1753). Emblica officinalis Gaertn. (1790). Local name: Amloki.

A bushy tree. On the hill top. Representative specimen: Runctia, 17.05.2007, Ershad Tutul 119 (DUH). 
Phyllanthus niruri L., Sp. Pl.: 981(1753). Local name: Bhuiamla.

An annual herb. On the hill slope. Representative specimen: Tawakocha, 28.10.2007, Ershad Tutul 245 (DUH).

Phyllanthus reticulatus Poir., Encycl. Meth. 5: 298 (1804). Cicca microcarpa Benth. (1861). Local name: Chitki.

A scandent shrub. Near the swamps. Representative specimen: Gazni, 16.05.2007, Ershad Tutul 42 (DUH).

Trewia nudiflora L., Sp. Pl.: 1193 (1753). Mallotus cardiophyllus Merr. (1912). Local name: Pitali.

A medium-sized tree. Near the swamps of forest. Representative specimen: Gazni, 17.05.2008, Ershad Tutul 342 (DUH).

\section{Rhamnaceae}

Gouania tiliaefolia Lamk., Encyl. 3: 5 (1789). Gouania leptostachya DC. (1825).

A large climber with lateral tendrils. On the edge of forest. Representative specimen: Gazni, 16.05.2008, Ershad Tutul 334 (DUH).

Ziziphus oenoplia (L.) Mill., Gard. Dict. ed. 8. no. 3 (1768). Rhamnus oenoplia L. (1762). Local name: Bonboroi.

A prickly shrub. On the hill slope. Representative specimen: Runctia, 17.05.2007, Ershad Tutul 115 (DUH).

\section{Leeaceae}

Leea crispa L., Mant. 1: 124 (1767). Local name: Banchalita.

A gregarious undershrub to shrub. On the hill slope. Representative specimen: Gazni, 18.05.2007, Ershad Tutul 181 (DUH).

Leea indica Merr., Philipp. J. Sci. Bot. 14: 245 (1914). Leea sambucina Willd. (1797). Local name: Kukura.

A much-branched shrub. On the hill slope. Representative specimen: Gazni, 17.05.2008, Ershad Tutul 353 (DUH).

\section{Vitaceae}

Cissus adnata Roxb., Fl. Ind. ed. Carey 1: 405 (1820). Cissus assamica (Lawson) Craib var. pilosissima Gagnep. (1911). Local name: Alianga lata.

A woody climber. On the hill slope. Representative specimen: Gazni, 18.05.2007, Ershad Tutul 154 (DUH).

Cissus repens Lamk., Encycl. Math. Bot. 1: 31 (1783). Vitis repens (Lamk.) Wight \& Arn. (1834). Local name: Marmaria lata. 
A large climber. On the hill slope. Representative specimen: Gazni, 17.05.2008, Ershad Tutul 383 (DUH).

Cayratia trifolia (L.) Domin, Biblioth. Bot. 89: 371 (1927). Vitis carnosa (Lamk.) Wall. ex Wight \& Arn. (1834).

A climber with swollen rootstock. On the hill slope. Representative specimen: Gazni, 17.05.2008, Ershad Tutul 377 (DUH).

\section{Burseraceae}

Protium serratum (Wall. ex Coelbr.) Engl. in DC., Monogr. Phan. 4: 88 (1883). Bursera serrata Wall. ex Colebr. (1827). Local name: Gutgutia.

An evergreen or semi-deciduous tree. On the hill slope. Representative specimen: Runctia, 17.05.2007, Ershad Tutul 146 (DUH).

\section{Anacardiaceae}

Anacardium occidentale L., Sp. Pl. 1: 383 (1753). Local name: Kaju badam.

An evergreen tree. On the top of hill, also planted. Representative specimen: Gazni, 17.05.2008, Ershad Tutul 381 (DUH).

Spondias pinnata (L. f.) Kurz, Pegu Rep. A. : 44 (1875). Spondias mangifera Willd. (1799). Local name: Amra.

A deciduous, glabrous tree. On the hill slope, planted also. Representative specimen: Gazni, 16.05.2007, Ershad Tutul 68 (DUH).

\section{Meliaceae}

Aglaia spectabilis (Miq.) Jain \& Bennet, Ind. J. For. 9: 271 (1987). Amoora ridleyi King (1895).

A medium-sized evergreen tree. On the hill slope. Representative specimen: Gazni, 16.05.2007, Ershad Tutul 102 (DUH).

Aphanamixis polystachya (Wall.) R. N. Parker, Ind. For. 57: 486 (1931). Amoora rohituka (Roxb.) Wight \& Arn. (1833). Local name: Pitraj.

A large tree. On the top of hill. Representative specimen: Gazni, 16.05.2007, Ershad Tutul 106 (DUH).

Azadirachta indica A. Juss., Mem. Mus. Hist. Nat. Paris 19: 221, t. 13 (1832). Melia indica (A. Juss.) Brandis (1874). Local name: Nim.

A medium-sized tree. On the hill slope and edges of forest. Representative specimen: Tawakocha, 28.10.2007, Ershad Tutul 279 (DUH).

Melia azedarach L., Sp. Pl. 1: 384 (1753). Azedarach sempervirens (L.) O. Kuntze (1891). Local name: Gora nim. 
A medium-sized tree. On the hill slope. Representative specimen: Gazni, 16.05.2007, Ershad Tutul 76 (DUH).

Toona ciliata M. Roem., Synops. Monogr. 1: 139 (1846). Toona ciliata M. Roem. var. parviflora (Benth.) Bahadur (1988). Local name: Rangil.

A medium to large tree. On the hill slope. Representative specimen: Tawakocha, 28.10.2007, Ershad Tutul 284 (DUH).

\section{Rutaceae}

Clausena suffruticosa (Roxb.) Wight \& Arn., Prodr.: 96 (1834). Amyris suffruticosa Roxb. (1832). Local name: Kalomaricha.

A small shrub. On the forest edges. Representative specimen: Gazni, 16.05.2007, Ershad Tutul 90 (DUH).

Glycosmis pentaphylla (Retz.) A. DC., Prodr. 1: 538 (1824). Glycosmis arborea (Roxb.) A. DC. (1824). Local name: Matkila.

An evergreen shrub. On the forest edges. Representative specimen: Tawakocha, 28.10.2007, Ershad Tutul 292 (DUH).

Micromelum minutum (J. G. Forster) Wight \& Arn., Prodr. Fl. Ind. Or.: 448, 468 (1834). Micromelum compressum (Blanco) Merr. (1918).

An unarmed shrub. On the hill slope. Representative specimen: Gazni, 18.05.2007, Ershad Tutul 187 (DUH).

Zanthoxylum rhetsa (Roxb.) DC., Prodr. 1: 728 (1825). Zanthoxylum budrunga (Roxb.) DC. (1824). Local name: Bajna.

A medium-sized deciduous, spiny tree. On the forest edges. Representative specimen: Gazni, 17.05.2008, Ershad Tutul 372 (DUH).

\section{Oxalidaceae}

Biophytum sensitivum (L.) DC., Prodr. 1: 690 (1824). Oxalis sensitiva L. (1753).

An annual herb. On moist fallow land and hill slope. Representative specimen: Gazni, 18.05.2007, Ershad Tutul 240 (DUH).

\section{Araliaceae}

Schefflera bengalensis Gamble, Kew Bull.: 229 (1919).

A climbing shrub. On bushy areas of forest. Representative specimen: Gazni, 16.05.2007, Ershad Tutul 77 (DUH).

Schefflera elliptica (Blume) Harms in Engl. \& Prantl, Pflanzenfam. 3(8): 39 (1894). Sciadophyllum elliptica Blume (1826).

A woody climber, often epiphytic. On the hill slope. Representative specimen: Tawakocha, 28.10.2007, Ershad Tutul 293 (DUH). 
Trevesia palmata (Roxb.) Vis., Mem. Acad. Torin. 2, 4: 262 (1842). Gastonia palmata Roxb. (1824). Local name: Argoja.

An erect shrub. On shady areas of forest. Representative specimen: Gazni, 17.05.2008, Ershad Tutul 375 (DUH).

\section{Apiaceae}

Centella asiatica (L.) Urban in Mart., Fl. Braz. 11(1): 187 (1879). Hydrocotyle asiatica L. (1753). Local name: Thankuni.

A perennial herb. On wet moist lands. Representative specimen: Tawakocha, 28.10.2007, Ershad Tutul 295 (DUH).

\section{Apocynaceae}

Alstonia scholaris (L.) R. Br., Mem. Wern. Nat. Hist. Soc. 1: 76 (1811). Nerium tinctorium Perr. (1824). Local name: Chhatim.

A tall tree. On the hill top. Representative specimen: Gazni, 17.05.2008, Ershad Tutul 392 (DUH).

Holarrhena antidysenterica (L.) Wall. ex Decne., Prodr. 8: 413 (1844). Holarrhena pubescens Wall. ex G. Don (1837). Local name: Kurchi.

A shrub to tree. Common on hill top and slopes. Representative specimen: Tawakocha, 28.10.2007, Ershad Tutul 261 (DUH).

Ichnocarpus frutescens (L.) R. Br., Mem. Wern. Soc. 1: 62 (1811). Ichnocarpus volubilis Merr. (1922). Local name: Dudhlata.

A large climber. On moist lands of forest. Representative specimen: Gazni, 16.05.2007, Ershad Tutul 105 (DUH).

Rauvolfia serpentina (L.) Benth. ex Kurz, Forest Fl. Brit. Burm. 2: 171 (1877). Tabernaemontana cylindracea Wall. (1829). Local name: Sarpogandha.

A woody herb. On the edges of forest. Representative specimen: Runctia, 17.05.2007, Ershad Tutul 136 (DUH).

\section{Solanaceae}

Solanum sisymbrifolium Lamk., Illus. 2: 25 (1797). Solanum balbisii Dunal (1825). Local name: Kata begun.

A very prickly herb. On the hill slope. Representative specimen: Gazni, 15.05.2007, Ershad Tutul 28 (DUH).

\section{Convolvulaceae}

Argyreia roxburghii Choisy, Mem. Soc. Phys. Genev. 6: 419 (1833).

A large climber. Near the swamps. Representative specimen: Gazni, 16.05.2008, Ershad Tutul 324 (DUH). 
Ipomoea fistulosa Mart. ex Choisy in DC., Prodr. 9: 349 (1845). Ipomoea crassicaulis (Benth.) B. L. Robinson (1916). Local name: Dhol kolmi.

An erect shrub. Near the swamps. Representative specimen: Tawakocha, 28.10.2007, Ershad Tutul 250 (DUH).

Merremia umbellata (L.) Hallier f., Bot. Jahrb. 16: 552 (1893). Ipomoea cymosa (Desr.) (1819). Local name: Sada kolmi.

A climber. On the forest edges. Representative specimen: Gazni, 16.05.2007, Ershad Tutul 46 (DUH).

Merremia vitifolia (Burm. f.) Hallier f., Bot. Jahrb. 16: 552 (1893). Ipomoea vitifolius Burm. f. (1768).

A large twiner. On the hill slope. Representative specimen: Gazni, 16.05.2007, Ershad Tutul 100 (DUH).

\section{Boraginaceae}

Heliotropium indicum L., Sp. Pl. 1: 130 (1753). Heliotropium velutinum DC. (1845). Local name: Hatisur.

An annual herb. Near swamps and on the edges of forest. Representative specimen: Gazni, 17.05.2008, Ershad Tutul 359 (DUH).

\section{Verbenaceae}

Callicarpa arborea Roxb., Fl. Ind. 1: 405 (1820). Local name: Bormala.

A deciduous tree. On the hill top. Representative specimen: Gazni, 16.05.2007, Ershad Tutul 111 (DUH).

Clerodendrum viscosum Vent., Jard. Malm. 1: 25 (1803). Clerodendron pubescens Wall. ex Walp. (1843). Local name: Vat.

A woody herb. Most common plant in the forest. Representative specimen: Gazni, 15.05.2007, Ershad Tutul 03 (DUH).

Tectona grandis L. f., Suppl.: 151 (1781). Theka grandis (L. f.) Lamk. (1793). Local name: Segun.

A large deciduous tree. On the hill top. Representative specimen: Gazni, 15.05.2007, Ershad Tutul 19 (DUH).

Vitex negundo L., Sp. Pl.: 638 (1753). Vitex paniculata Lamk. (1788). Local name: Nishinda.

A large shrub. On the edges of forest. Representative specimen: Gazni, 18.05.2007, Ershad Tutul 225 (DUH).

Vitex peduncularis Wall. ex Schauer in A. DC., Prodr. 11: 687 (1847). Vitex alata Roxb. (1832). Local name: Awal. 
A moderate-sized tree. On the hill slope. Representative specimen: Gazni, 18.05.2007, Ershad Tutul 188 (DUH).

Vitex trifolia L. f., Suppl.: 293 (1781). Vitex triphylla Royle (1839).

An aromatic shrub. On the hill slope. Representative specimen: Gazni, 17.05.2008, Ershad Tutul 377 (DUH).

\section{Lamiaceae}

Anisomeles indica (L.) O. Kuntze, Rev. Gen.: 512 (1891). Anisomeles ovata R. Br. (1811). Local name: Gobura.

An annual herb. On the forest edges. Representative specimen: Tawakocha, 28.10.2007, Ershad Tutul 247 (DUH).

Leucas aspera (Willd.) Link, Enum. Hort. Berol. 2: 113 (1822). Phlomis aspera Willd. (1809). Local name: Dandakalos.

An annual herb. On the forest edges. Representative specimen: Runctia, 17.05.2007, Ershad Tutul 125 (DUH).

Leucas indica (L.) R. Br. ex Vatke in Oesterr., B. Zeits. 25: 95 (1875). Leucas lavandulaefolia Smith (1819). Local name: Shetodron.

An annual herb. On the forest edges. Representative specimen: Gazni, 17.05.2008, Ershad Tutul 348 (DUH).

\section{Scrophulariaceae}

Limnophila indica (L.) Druce, Rep. Bot. Soc. Exch. Club Br. Isles 3: 420 (1914). Limnophila gratioloides R. Br. (1810).

An aquatic herb. Near the swamps of the forest. Representative specimen: Tawakocha, 28.10.2007, Ershad Tutul 283 (DUH).

Lindernia ciliata (Colsm.) Pennell, Brittonia 2: 182 (1936). Lindernia serrata (Roxb.) Muell.-Arg. (1882). Local name: Bhui.

A very small, annual herb. Near the swamps of the forest. Representative specimen: Tawakocha, 28.10.2007, Ershad Tutul 243 (DUH).

Scoparia dulcis L., Sp. Pl.: 116 (1753). Scoparia grandiflora Nash (1896). Local name: Bondhone. A perennial herb. On the edges and near the swamps of the forest. Representative specimen: Gazni, 15.05.2007, Ershad Tutul 31 (DUH).

\section{Acanthaceae}

Justicia gendarussa Burm. f., Fl. Ind.: 10 (1768). Gendarussa vulgaris Nees (1832). Local name: Jagatmardan.

An undershrub. On hill slope. Representative specimen: Tawakocha, 28.10.2007, Ershad Tutul 272 (DUH). 
Thunbergia grandiflora (Roxb. ex Rottler) Roxb., Bot. Reg. 6: t. 495 (1820). Thunbergia cordifolia Nees (1847). Local name: Nillata.

A large climber. Epiphyte on Lagerstroemia spices. Representative specimen: Gazni, 16.05.2007, Ershad Tutul 99 (DUH).

\section{Bignoniaceae}

Oroxylum indicum (L.) Kurz, For. Fl. Brit. Burm. 2: 237 (1877). Bignonia indica L. (1753). Local name: Kanaidingi.

A small to medium-sized tree. On the hill top and slope. Representative specimen: Gazni, 17.05.2008, Ershad Tutul 393 (DUH).

\section{Rubiaceae}

Catunaregam spinosa (Thunb.) Tirveng., Bull. Mus. Hist. Nat. (Paris) ser. 3, 35: 13 (1978). Randia spinosa (Thunb.) Bl. (1826). Local name: Mankanta.

A spiny shrub. On the hill slope. Representative specimen: Gazni, 15.05.2007, Ershad Tutul 32 (DUH).

Haldina cordifolia (Roxb.) Ridsdale, Blumea 24: 361 (1978). Adina cordifolia Hook. f. ex Brandis (1874). Local name: Keli kadam.

A large deciduous tree. On the top of hill. Representative specimen: Gazni, 16.05.2007, Ershad Tutul 58 (DUH).

Ixora javanica DC., Prodr. 4: 487 (1830). Local name: Rangan.

A shrub. On the forest bed. Representative specimen: Gazni, 18.05.2007, Ershad Tutul 230 (DUH).

Meyna spinosa Roxb. ex Link, Jahrb. Gewachsk 1(3): 32 (1820). Meyna laxiflora Robyns. (1828).

An armed tree. On hill slopes. Representative specimen: Gazni, 18.05.2007, Ershad Tutul 191 (DUH).

Morinda angustifolia Roxb., Fl. Ind. 1: 547 (1820). Local name: Bonamali.

An evergreen shrub or small tree. On the forest edges. Representative specimen: Runctia, 17.05.2007, Ershad Tutul 138 (DUH).

Mussaenda frondosa L., Sp. Pl.: 177 (1753). Mussaenda macrophylla sensu Kurz (1877) non Wall. Local name: Nagaballi.

A scandent shrub. On the hill slopes. Representative specimen: Gazni, 28.10.2007, Ershad Tutul 297 (DUH).

Mussaenda macrophylla Wall. in Roxb., Fl. Ind. 2: 228 (1824). Mussaenda calycina Wall. ex Kurz (1874).

A shrub. On the hill slope. Representative specimen: Gazni, 16.05.2007, Ershad Tutul 80 (DUH). 
Mussaenda roxburghii Hook. f., Fl. Brit. Ind. 3: 87 (1880). Mussaenda corymbosa Kurz (1874).

An erect or suberect shrub. On the hill slope. Representative specimen: Gazni, 16.05.2007, Ershad Tutul 81 (DUH).

Neolamarckia cadamba (Roxb.) Bosser, Bull. Mus. Hist. Nat. (Paris) 4, ser. 6, sec. B, 3: 247 (1984). Anthocephalus cadamba (Roxb.) Miq. (1856). Local name: Kadam.

A large deciduous tree. On the hill slope. Representative specimen: Gazni, 16.05.2008, Ershad Tutul 329 (DUH).

Oxyceros kunstleri (King \& Gamble) Tirveng., Nordic J. Bot. 3(4): 466 (1983). Randia scandens DC. (1830).

A rambling shrub. On the shady hill slope. Representative specimen: Gazni, 18.05.2007, Ershad Tutul 152 (DUH).

Paederia foetida L., Mant. 1: 52 (1767). Paederia tomentosa Blume. (1826). Local name: Gandhabadhuli.

A slender climber. On the hill slope. Representative specimen: Gazni, 28.10.2007, Ershad Tutul 268 (DUH).

Pavetta indica L., Sp. Pl.: 110 (1753). Ixora pavetta (L.) O. Kuntze (1891).

A large shrub. On the forest edges. Representative specimen: Gazni, 16.05.2007, Ershad Tutul 79 (DUH).

Pavetta naucleiflora R. Br. ex G. Don, Gen. Syst. 3: 575 (1834). Ixora naucleiflora Kurz (1877).

A large shrub. On the forest edges. Representative specimen: Gazni, 28.10.2007, Ershad Tutul 298 (DUH).

Spermacoce articularis L. f., Suppl. Pl.: 119 (1782). Borreria articularis (L. f.) Williams (1905). Local name: Madnabata kadu.

An annual herb. On the forest edges. Representative specimen: Tawakocha, 28.10.2007, Ershad Tutul 285 (DUH).

Spermacoce latifolia Aublet, Hist. Pl. Guiane Frtan. 1: 55, t. 19, f. 1 (1775). Borreria latifolia (Aublet) K. Schum. (1888).

An annual or perennial herb. Near the swamps. Representative specimen: Gazni, 16.05.2007, Ershad Tutul 92 (DUH).

\section{Asteraceae}

Ageratum conyzoides L., Sp. Pl.: 839 (1753). Local name: Fulkuri.

An annual herb. On the hill slope and edges. Representative specimen: Gazni, 18.05.2007, Ershad Tutul 169 (DUH). 
Blumea lacera (Burm. f.) DC. in Wight, Contrib. Bot. Ind.: 14 (1834). Conyza lacera Burm. f. (1768). Local name: Kukursunga.

An annual herb. On the forest edges. Representative specimen: Gazni, 15.05.2007, Ershad Tutul 18 (DUH).

Chromolaena odorata (L.) King \& Robinson, Phytologia 20: 204 (1970). Eupatorium odoratum L. (1759). Local name: Motmoti.

An erect, annual herb or undershrub. Very common on the edges of forest. Representative specimen: Gazni, 18.05.2007, Ershad Tutul 156 (DUH).

Elephantopus scaber L., Sp. Pl.: 814 (1753). Elephantopus scaber L. var. typicus Koster (1935).

A perennial herb. On the forest edges and forest floors. Representative specimen: Gazni, 16.05.2007, Ershad Tutul 57 (DUH).

Mikania cordata (Burm. f.) Robinson, Contr. Gray Herb. 104: 65 (1934). Mikania volubilis Willd. (1803). Local name: Assamlata.

A twining, perennial herb. On the edges of forest. Representative specimen: Gazni, 28.10.2007, Ershad Tutul 266 (DUH).

Vernonia cinerea (L.) Less., Linnaea 4(1): 291 (1829). Conyza cinera L. (1753). Local name: Kuksim.

An annual herb. On the hill slope and the edges of forest. Representative specimen: Gazni, 15.05.2007, Ershad Tutul 23 (DUH).

Vernonia extensa DC., Prodr. 5: 33 (1836). Vernonia cylindriceps C. B. Clarke (1876).

An annual herb. On the forest edges. Representative specimen: Gazni, 28.10.2007, Ershad Tutul 299 (DUH).

\section{References}

Cronquist, A. 1981. An integrated system of classification of flowering plants. Columbia University Press, New York. 1262 pp.

Dassanayake, M.D. and Fosberg, F.R. (eds.). 1980-1985. A Revised Handbook to the Flora of Ceylon, Vols. 1-5. Amerind Publishing Co. Pvt. Ltd., New Delhi.

Hajra, P.K., Nair, V.J. and Daniel, P. (eds.). 1997. Flora of India, Vol. 4. Botanical Survey of India, Calcutta. $561 \mathrm{pp}$.

Hassan, M.A. 1996. Three new combinations in Persicaria Mill. (Polygonaceae) from Bangladesh. Bangladesh J. Plant Taxon. 3(1): 81-83.

Hooker, J.D. 1872-1888. The Flora of British India, Vols. 1-5 (Ind. Repr. 1973). Bishen Singh Mahendra Pal Singh, Dehra Dun, India.

Kanjilal, U.N., Kanjilal, P.C. and Das, A. 1934. Flora of Assam, Vol. 1. A Von Book Company, Delhi, India. $386 \mathrm{pp}$.

Kanjilal, U.N., Kanjilal, P.C. and Das, A. 1938. Flora of Assam, Vol. 2. A Von Book Company, Delhi, India. 409 pp. 
Kanjilal, U.N., Das, A., Kanjilal, P.C. and De, R.N. 1939. Flora of Assam, Vol. 3. A Von Book Company, Delhi, India. 578 pp.

Kanjilal, U.N., Kanjilal, P.C., De, R.N. and Das, A. 1940. Flora of Assam, Vol. 4. A Von Book Company, Delhi, India. 377 pp.

Khan, M.S. (ed.). 1972-1987. Flora of Bangladesh, Nos. 1-39. Bangladesh National Herbarium, BARC, Dhaka.

Khan, M.S. and Rahman, M.M. (eds.). 1989-2002. Flora of Bangladesh, Nos. 40-53. Bangladesh National Herbarium, Dhaka.

Prain, D. 1903. Bengal Plants. Vols. 1 \& 2 (Ind. Repr. 1981). Bishen Singh Mahendra Pal Singh, Dehra Dun, India.

Singh, N.P., Vohra, J.N., Hajra, P.K. and Singh, D.K. (eds.). 2000. Flora of India, Vol. 5. Botanical Survey of India, Calcutta. 577 pp.

Tutul, E., Uddin, M.Z., Rahman, M.O. and Hassan, M.A. 2009. Angiospermic flora of Runctia sal forest, Bangladesh. I. Liliopsida (Monocots). Bangladesh J. Plant Taxon. 16(1): 83-90.

(Manuscript received on 16 July 2009; revised on 29 August 2009) 